\title{
Alteration of TGFB1, GDF9, and BMPR2 gene expression in preantral follicles of an estradiol valerate-induced polycystic ovary mouse model can lead to anovulation, polycystic morphology, obesity, and absence of hyperandrogenism
}

\author{
Reza Asghari ${ }^{1}$, Vahid Shokri-Asl ${ }^{2}$, Hanieh Rezaei ${ }^{3}$, Mahmood Tavallaie ${ }^{4}$, Mostafa Khafaei $^{4}$, Amir Abdolmaleki ${ }^{5}$, \\ Abbas Majdi Seghinsara'
}

'Department of Anatomical Sciences and Histology, Faculty of Medicine, Tabriz University of Medical Sciences, Tabriz; ${ }^{2}$ Department of Reproductive Biology, Faculty of Advanced Medical Sciences, Tabriz University of Medical Sciences, Tabriz; ${ }^{3}$ Member of Research Committee, Medical School, Hamedan University of Medical Sciences, Hamedan; ${ }^{4}$ Human Genetic Research Center, Baqiyatallah University of Medical Sciences, Tehran; ${ }^{5}$ Department of Anatomical Sciences, Medical School, Kermanshah University of Medical Sciences, Kermanshah, Iran

Objective: In humans, polycystic ovary syndrome (PCOS) is an androgen-dependent ovarian disorder. Aberrant gene expression in folliculogenesis can arrest the transition of preantral to antral follicles, leading to PCOS. We explored the possible role of altered gene expression in preantral follicles of estradiol valerate (EV) induced polycystic ovaries (PCO) in a mouse model.

Methods: Twenty female balb/c mice ( 8 weeks, $20.0 \pm 1.5 \mathrm{~g}$ ) were grouped into control and PCO groups. PCO was induced by intramuscular EV injection. After 8 weeks, the animals were killed by cervical dislocation. Blood serum (for hormonal assessments using the enzyme-linked immunosorbent assay technique) was aspirated, and ovaries (the right ovary for histological examinations and the left for quantitative real-time polymerase) were dissected.

Results: Compared to the control group, the PCO group showed significantly lower values for the mean body weight, number of preantral and antral follicles, serum levels of estradiol, luteinizing hormone, testosterone, and follicle-stimulating hormone, and gene expression of TGFB1, GDF9 and BMPR2 $(p<0.05)$. Serum progesterone levels were significantly higher in the PCO animals than in the control group $(p<0.05)$. No significant between-group differences $(p>0.05)$ were found in BMP6 or BMP15 expression.

Conclusion: In animals with EV-induced PCO, the preantral follicles did not develop into antral follicles. In this mouse model, the gene expression of TGFB1, GDF9, and BMPR2 was lower in preantral follicles, which is probably related to the pathologic conditions of PCO. Hypoandrogenism was also detected in this EV-induced murine PCO model.

Keywords: Bone morphogenetic protein 6; Bone morphogenetic protein 15; Bone morphogenetic protein receptor II; Folliculogenesis; Growth differentiation factor 9; Multicystic morphology of ovary; Polycystic ovary syndrome; Transforming growth factor- $\beta 1$

Received:September 25, 2020 · Revised: April 3, 2021 · Accepted: May 12, 2021 Corresponding author: Abbas Majdi Seghinsara

Department of Anatomical Sciences and Histology, Faculty of Medicine, Tabriz University of Medical Sciences, Central Building, 3rd Floor, Tabriz University Main Street, Tabriz 5166616471, Iran

Tel: +98-9633355921 Fax:+98-33359680 E-mail: abas.majdii57@gmail.com

This is an Open Access article distributed under the terms of the Creative Commons Attribution Non-Commercial License (http://creativecommons.org/licenses/by-nc/4.0/) which permits unrestricted non-commercial use, distribution, and reproduction in any medium, provided the original work is properly cited.

\section{Introduction}

Polycystic ovary syndrome (PCOS) is known to be the most common cause of female infertility in reproductive-age women (18-44 years old). In the human body, the initial basis of PCOS relates to the presence of male sex hormones (hyperandrogenism) at high levels; thus, it is sometimes considered to be an endocrine disorder [1]. 
Higher androgen levels can lead to hyperandrogenism, anovulation, follicular cysts, menstrual cycle irregularities, and eventually, female infertility $[2,3]$. PCOS has a primarily genetic basis. Ovarian follicles are the smallest affected unit in folliculogenesis. Each follicle contains a single central oocyte, granulosa cells, and an outer structure of connective tissue named the theca. A fluid-filled cavity formed adjacent to the oocyte is known as an antrum, and a follicle with an antrum is designated as an antral follicle. A follicle with no antrum is referred to as a preantral follicle. The transition of preantral to antral follicles is a normal and routine procedure known as folliculogenesis. Alterations of the genes involved in this process can arrest the transition from the preantral to antral stages, which is a defining feature of PCOS pathophysiology [4].

Folliculogenesis is fundamentally based on the secretion of various types of growth factors (GFs) by ovarian somatic cells [5]. Two major types of GFs are growth differentiation factor-9 (GDF9) and transforming growth factor-beta1 (TGF- $\beta 1$ ) $[6,7]$, which are necessary for folliculogenesis and cell proliferation, respectively. Bone morphogenetic protein (BMP)-15 is involved in folliculogenesis, including the growth and maturation of ovarian follicles, regulation of the sensitivity of granulosa cells to follicle-stimulating hormone (FSH), determination of the number of ovulated follicles, and prevention of granulosa cells from apoptosis. As such, BMP15 is a crucial agent for the initiation of folliculogenesis [8]. It is released from the oocyte into the surrounding granulosa tissue to promote granulosa cell proliferation [9]. BMP receptor II (BMPR2 is expressed in granulosa cells. BMPR2 is a crucial receptor for BMP15 and GDF9. These 2 signaling molecules and their receptors have impressive effects on follicular development and ovulation. BMP6 is abundant in granulosa cells, and serves as an important mediator to support follicular growth [10].

The physiological functions of TGF- $\beta 1$, BMP6, BMP15, GDF9, and BMPR2 in ovaries have been confirmed in previous research, but no comprehensive investigations exist regarding the altered expression of these genes in preantral follicles in PCOS; this is of particular importance because the expression of the genes involved in folliculogenesis depends on follicular stage [11]. Thus, this study aimed to investigate the role of related gene expression in preantral follicles following morphological induction of polycystic ovaries (PCO) in mice.

\section{Methods}

\section{Animals}

Twenty balb/c female mice (20.0 $\pm 1.5 \mathrm{~g}$, 8 weeks old) were obtained from the university's home. All standard conditions for animal keeping were provided, including food pellets (Livestock and Poultry Feed Company, Tehran, Iran), free access to water, transparent and separated cages, equilibrium of the environmental temperature $\left(22^{\circ} \mathrm{C}\right)$, humidity of $55 \% \pm 3 \%$, and a 12 -hour light/12-hour dark photo-cycle.

\section{Ethical considerations}

All animals were treated in accordance with guidelines of the $\mathrm{Na}$ tional Institute of Health for the Care and Use of Laboratory Animals approved by the Research Deputy based on the WMA Declaration of Helsinki (ethics approval No. IR.TBZMED.REC. 1396.346).

\section{Study groups}

The animals were randomly divided into control and PCO groups ( $n=10$ each). In the control group, the animals received an intramuscular injection of sesame oil $(0.1 \mathrm{~mL})$, and the animals in the PCO group received PCO induction by intramuscular injections of estradiol valerate (EV) [12].

\section{Follicular classification}

According to the routine protocol, the classification of follicles was applied based on the morphological features, as follows: primary ovarian follicles (with a single layer of cuboidal granulosa cells), preantral follicles (containing one or two small spaces filled by follicular fluid), antral follicles (consisting of a single large antral space), and preovulatory follicles (a rim of cumulus cells around the oocyte suspended in a large space) [13].

\section{PCOS induction}

$\mathrm{EV}$ is an exogenous chemical agent that can completely mimic PCO morphologically. EV (Sigma Chemical Co., St. Louis, MO, USA) was injected intramuscularly on 2 consecutive days (each injection comprised $0.2 \mathrm{~mL}$ of EV dissolved in normal saline) to induce PCO in estrous cycle based on the morphological feature according to the Table 1.

\section{Animal dissection and tissue sampling}

Prior to the study and on the last day of the experiment, the animals were weighed. At 56 days (8 weeks) after PCO induction, the animals were killed by cervical dislocation. The subxiphoid approach was used for blood aspiration for future hormonal assays. Fifteen minutes later, the blood clot was centrifuged (4,000 rpm, $10 \mathrm{~min}$ utes). Midline laparotomy was then performed, and the ovaries were dissected from both sides of the body. The surrounding adipose tissue was separated, and the ovaries were quickly washed in normal saline. The right ovary was fixed in 10\% formalin for 3 days for morphological assessments, and the left ovary was used for gene expression analyses. 
Table 1. Cellular features of vaginal smear and determination of estrus cycle in mice

\begin{tabular}{lll}
\hline Stage of estrous cycle & \multicolumn{1}{c}{ Histological feature } & \multicolumn{1}{c}{ Physiological feature } \\
\hline Proestrous & Mostly nucleated epithelia cells+less cornified epithelial cells & $\begin{array}{l}\text { Initiation of follicular growth } \\
\text { No ability to receive pregnancy }\end{array}$ \\
& & Proliferation of vaginal epithelium \\
Estrous & High rate of cornified epithelial cells & Estrogen production \\
& & Highest level of estrogen \\
Metestrus & Cornified epithelial cells+leukocytes & Ovulation \\
Diestrus & Primarily leukocytes & Corpus luteum formation \\
\hline
\end{tabular}

\section{Separation of preantral and antral follicles}

Two microliters of alpha-minimum essential medium containing $3 \%$ fetal bovine serum was used as a suspension for mechanical tissue dissection of the ovaries. One G-needle tip attached to an insulin syringe was used for mechanical dissection using a stereomicroscope (Olympus, Tokyo, Japan). The studied follicles were morphologically multilayered preantral (1-8 $\mu \mathrm{m}$ in diameter) and antral follicles. The isolated follicles were located inside a micro-tube and stored at $-70^{\circ} \mathrm{C}$.

\section{Histological preparation and hematoxylin and eosin staining}

The right fixed ovary underwent routine tissue processing for hematoxylin and eosin (H\&E) staining. After tissue fixation (using 10\% formalin), dehydration (by different alcohol concentrations), and clarification (with xylol), the ovaries were embedded in paraffin. The slides were cut into 5 - $\mu \mathrm{m}$ sections and stained with $\mathrm{H} \& \mathrm{E}$ for morphological assessments of the follicles (Table 2).

\section{Hormone measurements}

To assess the levels of serum hormones, the aspirated blood samples were centrifuged $(4,000 \times g, 10$ minutes), and isolated serum was stored in a freezer $\left(-70^{\circ} \mathrm{C}\right)$ for biochemical analysis of luteinizing hormone (LH), FSH, progesterone, estradiol, and testosterone using enzyme-linked immunosorbent assays (Cayman Chemicals; Ann Arbor, MI, USA). In this test, specific monoclonal antibodies were used against an antigenic marker of the target hormone. One monoclonal antibody was used for solid-phase stabilization, and another anti-hormone antibody was used for conjugation with horseradish peroxidase. Upon adding a serum sample, the hormone reacted simultaneously with the above two antibodies and was sandwiched between the antibody bound to the solid phase and the conjugated antibody. After 4 minutes of incubation at room temperature to remove free labeled antibodies, the wells were rinsed with washing solution. After adding the substrate-dye solution (4 minutes of incubation), a blue color appeared. Color production was stopped by
Table 2. Morphological features of ovarian follicles

\begin{tabular}{ll}
\hline Type of ovarian follicles & \multicolumn{1}{c}{ Histological feature of ovaries } \\
\hline Primary & A single layer of cuboidal granulosa cells \\
Preantral & One or two small spaces filled by follicular fluid \\
Antral & A single large antral space \\
Preovulatory & A rim of cumulus cells around the oocyte \\
\hline
\end{tabular}

adding the stop solution and the blue color turned yellow. Finally, the color intensity was measured (at specific wavelengths, expressed in nanometers). The intensity of color was directly related to the concentration of hormones in serum.

\section{RNA extraction and CDNA formation}

Total RNA was extracted from the ovaries using the TRIzol reagent (Invitrogen, Carlsbad, CA, USA) based on the manufacturer's instructions (Cat No.11667165001; Roche, Basel, Switzerland). The quality and quantity of each sample were assessed by a NanoDrop ND-2000 Spectrophotometer (Thermo Fisher Scientific, Waltham, MA, USA) according to the optical density. The RNA integrity was confirmed by electrophoresis on 1\% agarose gel (Ultra-Pure TM Agarose, Invitrogen) stained with the Gel Red RNA stain detected under ultraviolet light. High-quality RNA samples with no degradation were stored at $-80^{\circ} \mathrm{C}$. Then, complementary DNA (CDNA) synthesis was performed using the Takara cDNA kit (Takara Cat No. 6130; Takara, Mountain View, CA, USA). The first-strand CDNA was synthesized using a cDNA synthesis kit (Thermo Fisher). Reverse transcription of $500 \mathrm{ng}$ of the total RNA extracted from all samples was performed using both random hexamer and oligo-dT primers.

\section{Quantitative real-time polymerase chain reaction}

Sense and antisense primers for the target and reference genes were designed using primer design software (National Center for Biotechnology Information, Primer-BLAST, https://www.ncbi.nlm. nih.gov/tools/primer-blast/). CRPL13A and GAPDH were used as reference genes for normalization. The primers were: GDF9 (forward [F]: CAGTCCACCTGGAGGCCTTTA, reverse [R]: GAGCGGATGGCTTTCTGC- 
CCT), BMP15 (F: TGGGCCTCTTCTCCTCGGTTA, R: AATCTTCTGGGCCAAAGCGAG), TGFB1 (F: ACTTCTTGGCACTGCGCTGTC, R: GAACGCCGGGTAGCGATCGAG), BMPR2 (F: AGGAGCTGTGAACCTGAGGGA, R: TCACCTGGGAAGAGGTCTGTA), and BMP6 (F: GGCCTTCTTCAAGGTGAGCGA, R: TAGTCTGAAGAACCGGAGCCC). The Quantitative polymerase chain reaction was performed using the Ampliqon Kit by Rotor-Gene 6000 system (Corbett Research, Sydney, Australia). The cycling conditions were $95^{\circ} \mathrm{C}$ (15 minutes), 40 cycles of amplification $\left(95^{\circ} \mathrm{C}, 60^{\circ} \mathrm{C}\right.$, and $72^{\circ} \mathrm{C} ; 40$ seconds each), followed by a final extension of $72^{\circ} \mathrm{C}$ (5 minutes). Finally, melting was carried out for 5 seconds at $60^{\circ} \mathrm{C}-95^{\circ} \mathrm{C}$ (with $0.5^{\circ} \mathrm{C}$ increments for each step). Relative quantification was applied using the Livak method and the samples' crossing point, the number of cycles completed, the efficiency of the reaction, and other values were used in the analysis for comparison and creating the ratios. Finally, the results were reported as normalized ratios (Table 3).

\section{Data analysis}

Data were analyzed using SPSS ver. 16 (SPSS Inc., Chicago, IL, USA). The normal distribution of the data was confirmed by the Kolmogorov-Smirnov test. The independent $t$-test and Tukey post-hoc test were used to compare differences among the groups. All data were presented as mean \pm standard deviation, and $p<0.05$ was considered as the significance level.

\section{Results}

\section{Total animal weight}

On day 56, the PCO group showed a significantly lower total weight $(26.3 \pm 1.2 \mathrm{~g})$ than the control group $(30.4 \pm 0.9 \mathrm{~g})(p<0.05)$.

\section{Number of preantral and antral follicles}

According to the histological data, the PCO group showed significantly lower mean numbers of antral follicles ( 6.5 in the control group vs. 5.7 in the PCO group) and preantral follicles (5.1 in the control group vs. 4.4 in the PCO group) $(p<0.05)$. However, no significant differences were detected in the number of primary follicles ( 6.5 in the control group vs. 5.7 in the PCO group) and pre-ovulatory follicles (0.26 in the control group vs. 0.08 in the PCO group).

Table 3. Nucleotide sequences for primers of studied genes

\begin{tabular}{lll}
\hline Gene & \multicolumn{1}{c}{ Forward } & \multicolumn{1}{c}{ Reverse } \\
\hline GDF9 & CAGTCCACCTGGAGGCCTTTA & GAGCGGATGGTTTCTGCCCT \\
BMP15 & TGGGCCTCTTCTCCTCGGTTA & AATCTTCTGGGCCAAAGCGAG \\
TGFb1 & ACTTCTTGGCACTGCGCTGTC & GAACGCCGGGTAGCGATCGAG \\
BMPR2 & AGGAGCTGTGAACCTGAGGGA & TCACCTGGGAAGAGGTCTGTA \\
BMP6 & GGCCTTCTTCAAGGTGAGCGA & TAGTCTGAAGAACCGGAGCCC \\
\hline
\end{tabular}

\section{Hormone assay}

In the PCO group, the levels of testosterone $(14.45 \mathrm{ng} / \mathrm{mL}$ in the control group vs. $5.13 \mathrm{ng} / \mathrm{mL}$ in the PCO group), estradiol ( $156.32 \mathrm{ng} / \mathrm{mL}$ in the control group vs. $52.14 \mathrm{ng} / \mathrm{mL}$ in the PCO group), LH (13.24 $\mathrm{ng} / \mathrm{mL}$ in the control group vs. $7.63 \mathrm{ng} / \mathrm{mL}$ in the PCO group), and FSH $(15.68 \mathrm{ng} / \mathrm{mL}$ in the control group vs. $8.85 \mathrm{ng} / \mathrm{mL}$ in the PCO group) were significantly lower $(p<0.05)$, whereas the level of progesterone $(19.46 \mathrm{ng} / \mathrm{mL}$ in the control group vs. $42.52 \mathrm{ng} / \mathrm{mL}$ in the PCO group) was significantly higher $(p<0.05)$ than in the control group (Figure 1).

\section{Histological assessment}

In the PCO group, the oocytes were wrinkled compared to those in the control group, and large cystic follicles with a narrow granulosa layer (1-3 cellular layers) along with a significant decreased number of preantral follicles were discovered. Furthermore, no corpus luteum was observed in the PCO group (Figure 2). In addition, a morphological assessment of the ovaries showed that the ovaries of EV-treated mice were smaller than those of the control group, with large cystic follicles and non-stromal hypertrophy.

\section{Gene expression}

The preantral follicles of the PCO group showed significantly lower expression of the following genes than the control group: GDF9 ( 0.0068 vs. 0.0042 in the control and PCO groups, respectively), TGFB1 ( 0.0045 vs. 0.0020 in the control and PCO groups, respectively), and BMPR2 (0.0075 vs. 0.0040 in the control and PCO groups, re-

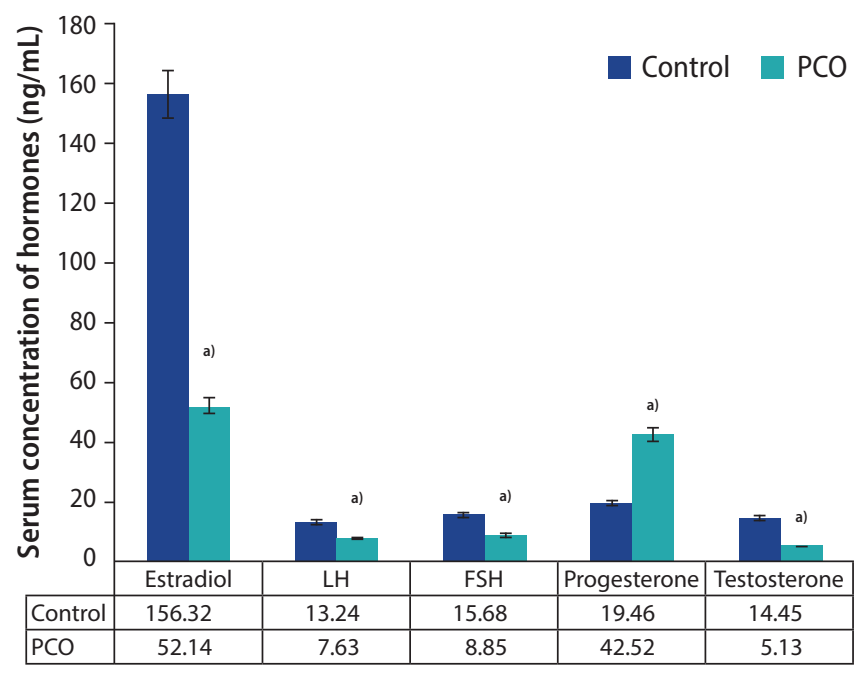

Hormone

Figure 1. Serum concentration of hormones $(\mathrm{ng} / \mathrm{mL})$. Values are presented as mean \pm standard deviation. $\mathrm{PCO}$, polycystic ovaries; $\mathrm{LH}$, luteinizing hormone; $\mathrm{FSH}$, follicle-stimulating hormone. ${ }^{\text {a) }}$ Significant differences $(p<0.05)$. 

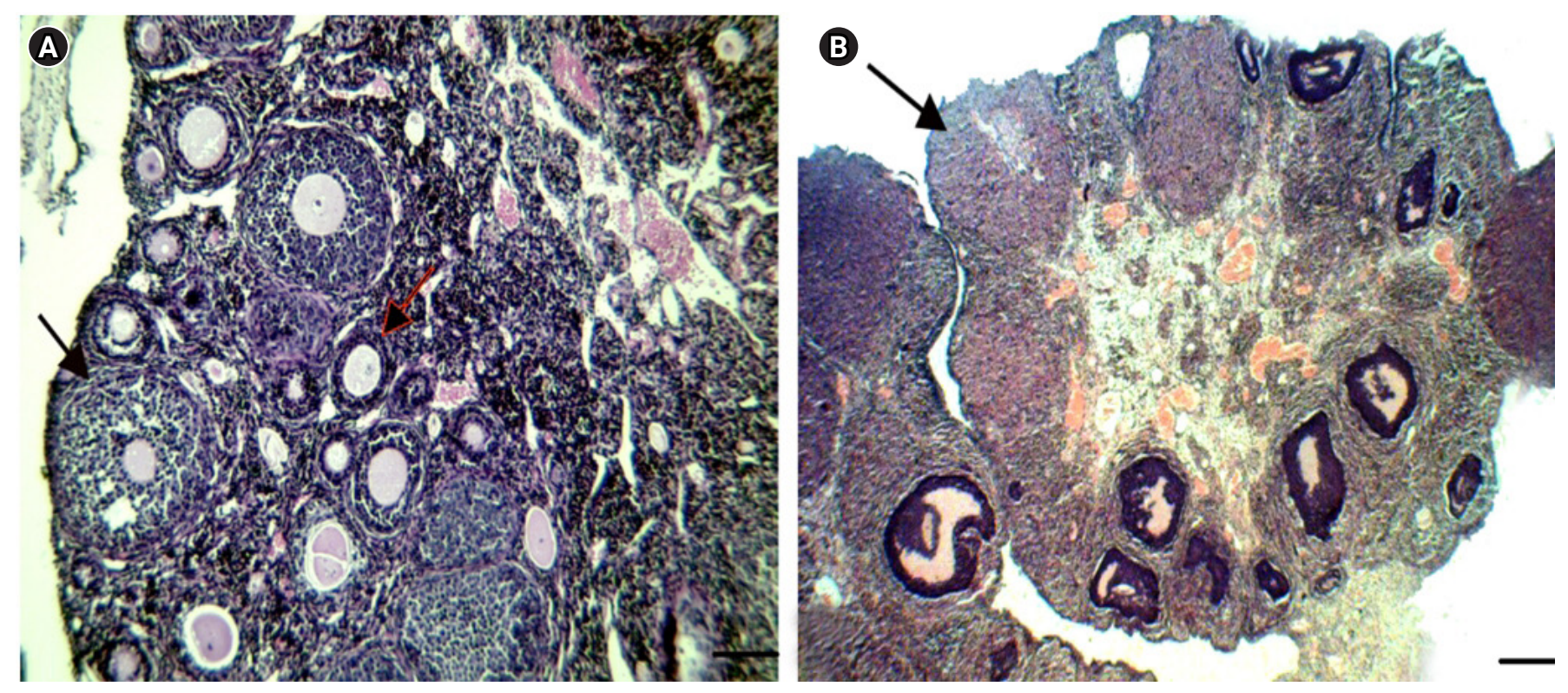

Figure 2. Histological photographs of the polycystic ovaries and control groups $(H \& E, \times 400)$. (A) Preantral (red arrow) and antral (black row) follicles (scale bar=200 $\mu \mathrm{m}$ ). (B) Corpus luteum (black arrow) in the control group (scale bar=100 $\mu \mathrm{m}$ ).

spectively) $(p<0.05)$. No significant differences were found in the expression of BMP6 (0.0048 vs. 0.0058 in the control and PCO groups, respectively) or BMP15 (0.0080 vs. 0.0075 in the control and PCO groups, respectively) in the preantral follicles $(p>0.05)$ (Figure 3 ).

\section{Discussion}

In adult women, folliculogenesis is a monthly process that can eventually lead to ovulation. A crucial stage in ovulation is the transition from preantral to antral follicles, a process that is regulated by the expression of important genes. Thus, any disruption of the preantral to antral transition can potentially arrest ovulation. In patients with PCOS, preantral follicles do not develop into the antral stage. Thus, in the present study, the role of the expression of genes (TGFB1, GDF9, BMP6, BMP15, and BMPR2) involved in the transition of preantral to antral follicles was investigated in an EV-induced PCO mouse model, and relationships with serum levels of sex hormones were explored biochemically, histologically, and genetically.

In PCOS-affected humans, hormonal and metabolic alterations in the ovaries are most likely associated with upregulation of PI3K-Akt [14] and increased levels of ERK activity [15] as intracellular and extracellular pathways, respectively. In animals, the morphological features of PCO are induced by EV injection, which cannot precisely mimic the clinical and molecular conditions of PCOS in humans in terms of metabolic parameters and hyperandrogenemia. Although there are crucial differences between PCO induction in animals (through EV injection) and humans morphologically, EV causes anovulation and multicystic ovaries with less stroma and more cystic

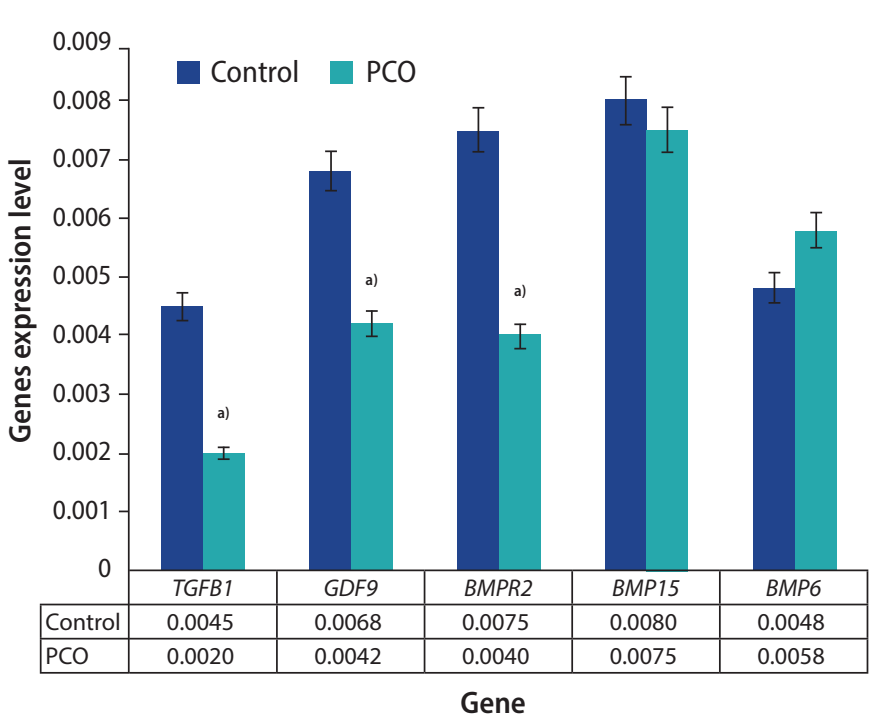

Figure 3. Comparison of gene expression levels in control preantral follicles and the polycystic ovaries (PCO) group. Values are presented as mean \pm standard deviation. ${ }^{\text {a) }}$ Significant differences $(p<0.05)$.

follicles. Thus, this drug was used as an exogenous chemical to model PCO in mice in the present study [12]. Furthermore, the ovulation processes in humans and rodents are mono-ovulation and poly-ovulation, respectively, suggesting that the FSH-dependent follicle selection process in rodents differs from that in humans $[16,17]$.

There are various types of PCO models in animals with different features and limitations. It is important to state that no single method of PCO induction in animal models exactly resembles human 
PCOS [18]. PCOS can be induced in different stages of animal life (prenatal, postnatal, prepubertal, and adolescence) with many inducing factors (environmental factors, genetic factors, and hormonal models); thus, this pathologic condition is known to be a multifactorial disease. Animal models of PCO are induced by various environmental factors, of which endocrine-disrupting chemicals (EDC) are the most common. Endocrine disrupting chemicals interfere with the hormonal balance, leading to PCO induction. Bisphenol-A (BPA), as a synthetic xenoestrogen, is an important EDC [18]. Neonatal exposure to BPA $(500 \mu \mathrm{g})$ caused an increased testosterone concentration in serum, which led to the induction of PCO-like features in the adulthood of animals, such as the formation of ovarian cysts [19]. BPA also induces PCOS-associated metabolic syndrome, which stimulates pancreatic beta-cells to produce insulin and acts as an obesogenic agent to release adiponectin from adipose tissue, which is known to be an agent for animal PCO induction [20]. Di-(2-ethylhexyl) phthalate (DEHP), another EDC, is one of the major environmental estrogens. DEHP administration ( $2 \mathrm{~g} / \mathrm{kg}$ body weight, 12 days) caused symptoms resembling PCO, including irregular cyclicity, anovulation, decreased serum estradiol, and polycystic follicle formation [21].

Tributyltin (TBT) is another endocrine disruptor with obesogenic properties. Since, obesity is associated with insulin resistance, which is one of the features of PCO, studies have focused on the development of PCO caused by TBT administration [22]. Since TBT causes irregular estrous cycles, hyperandrogenism, and obesity, this model exhibits most PCO metabolic features [23]. Hormonal interventions are another method to induce PCO in animal models. There are 3 main types of PCO animal models depending on the specific hormonal manipulation: androgen-induced, estrogen-related, and antiprogesterone-induced. Testosterone, dihydrotestosterone (DHT), dehydroepiandrosterone (DHEA), and esters of testosterone (testosterone propionate [TP]) are used to induce hyperandrogenism in animal PCO conditions. Some papers stated that hyperandrogenism could disrupt normal reproductive physiology, leading to PCO induction [24]. Postnatal intraperitoneal exposure of TP (10 mg/kg bw) showed the characteristic features of PCOS, such as irregular estrous cyclicity, elevated testosterone and insulin levels, the presence of cystic follicles with unaltered estradiol levels, reduced progesterone levels, arrested ovulation, and the absence of corpora lutea [25]. Various studies have reported that the administration of TP did not lead to an ovarian phenotype exactly resembling that observed in PCOS [18]. DHEA was the first agent used by scientists to induce PCO in animals due to the elevated levels of DHEA found in women with PCOS [26]. Subcutaneous injections of DHEA (60 mg/kg bw in 42-day-old rats for 20-30 days) led to an improved PCO model with increased body weight, irregular estrous cyclicity, and an increased number of ovarian cystic follicles [27]. A DHEA-induced PCO rodent model exhibited all key features similar to women with PCOS. DHT is a nonaromatizable androgen that cannot be converted into estrogen by the action of aromatase; thus, it enhances androgen potency. Exposure of prepubertal rats (7.5 mg) [28] and mice (2.5 mg) [29] to $\mathrm{DHT}$ (for 90 days) was found to be a precise model with features similar to those of women with PCOS, such as increased body weight and insulin resistance [30]. In women with PCOS, serum estrogen levels are elevated. Based on this evidence, estrogen or other forms of estrogen (e.g., EV or estradiol benzoate [EB]) are used to induce PCO in animal models. One-day-old neonatal rats treated with EB showed acyclicity and anovulation. However, the serum LH levels, as well as the ovarian weight, decreased, and elevated serum FSH levels were observed, unlike human PCOS conditions [31]. Rats exposed to EV (2 mg) failed to exhibit key features of PCOS, representing dissimilarities with human PCOS [32]. Prior studies suggested that, in estrogenic conditions, ovarian alterations similar to those observed in PCOS would not happen, and hormonal and metabolic disruptions were not parallel with PCOS women under exposure to estrogenic conditions. Among the animal models of $\mathrm{PCO}$, there are some important metabolic features and morphological characteristics, such as acyclicity, anovulation, hyperandrogenism, cystic follicles, insulin resistance, and obesity. Generally, DHEA is a chemical used to induce PCO with various serological, morphological, and metabolic alterations exactly resembling those found in human women with PCOS. Additionally, EV administration can induce acyclicity, anovulation, formation of cystic follicles, and obesity, with an approximate similarity to human PCOS. However, in EV-induced PCO animal models, there are no metabolic symptoms such as hyperandrogenism and insulin resistance [18]. It has finally been concluded that androgenized PCO mouse models are a better choice in terms of resembling human PCOS than models using estrogenic agents. Although we assessed preantral follicles in the present study, the EV-induced PCO mouse model did not exactly mimic human PCOS. The absence of hyperandrogenism and insulin resistance following the induction of PCO by EV constitute limitations of the present study. Due to these limitations of EV-induced PCO, DHEA is recommended for PCO induction in mice.

As shown by the data in the present study, weight loss occurred 56 days after PCO induction using intramuscular injections of EV. Although it is scientifically accepted that weight gain is common in PCOS patients [33], this study found weight loss in EV-induced PCO mice. This finding was also reported by lkeda et al. [34] following DHEA administration to animals. They found that a 1-month application of DHEA led to decreased total weight in PCO animals. Reduced levels of total body weight were also reported by Mesbah et al. [35] following EV administration. There are three main reasons underly- 
ing the biological process of weight loss in animals following intramuscular injections of EV: (1) the lipolytic characteristics of increased adrenal glucocorticoid levels; (2) neurological modifications in the hypothalamic paraventricular nucleus interfering with food intake; and (3) the activation of peripheral sympathetic neurons leading to metabolic activity promotion, increased fat consumption, and finally weight loss [32].

In morphological assessments, the numbers of preantral and antral follicles were lower in the PCO group. This phenomenon probably relates to the effects of $\mathrm{PCO}$ on inhibiting follicular development. Overall, although the ovaries in EV-treated mice were lower, the majority of the ovarian tissue comprised abnormal cystic follicles with large and filled follicles. Since the entirety of the ovaries included cystic follicles, the stromal tissue was smaller in PCO-affected animals.

In this study, changes in three major hormones secreted by PCO-affected ovaries (estradiol, progesterone, and testosterone) and two hormones secreted by the pituitary gland ( $\mathrm{LH}$ and FSH) were analyzed. The results showed significantly lower $\mathrm{LH}, \mathrm{FSH}$, testosterone, and estradiol levels in the PCO group than in the control group, but progesterone showed significantly higher levels. In humans, PCOS is characterized by hypersecretion of $\mathrm{LH}$. The LH surge in the primary stage can lead to ovulation, but chronic hypersecretion of $\mathrm{LH}$ can arrest follicular growth, leading to anovulation [36]. As we found in this investigation, PCO was characterized by a decreased number of preantral, antral, and pre-ovulatory follicles. As PCO has no effect on primary follicles, the number of primary follicles did not show significant differences. In an experimental study, Brawer et al. [37] assessed the development of PCOS in EV-injected rats, and reported decreased levels of $\mathrm{LH}$ and FSH. FSH stimulates the growth and recruitment of immature ovarian follicles in the ovary. Thus, reduced levels of FSH can eventually lead to arrest of the development of ovarian follicles [38]. Miri et al. [39] also assessed the effects of exercise intensity on sex hormones in female rats with EV-induced PCOS. They found that the PCOS animals showed decreased levels of testosterone in comparison with the control group. This finding is in line with our results, as we found hypoandrogenism. Parallel to our findings, Brawer et al. [37] also stated that the basal levels of LH and FSH decreased in EV-treated PCOS animals. An experimental study conducted by Pournaderi et al. [40] found the same results, indicating that after EV-induced PCOS, the animals presented decreased levels of LH, FSH, and estradiol and also found increased levels of progesterone in comparison with the control group. Venegas et al. [41] similarly found that increased serum levels of progesterone. Laird et al. [42], in an in vitro study, examined the role of testosterone on the transition of preantral follicles to the antral form. They concluded that testosterone could stimulate the growth of preantral follicles. However, in the present study, tes- tosterone levels were lower in the PCOS group, indicating arrest of the preantral to antral transition.

Among the genes affecting the ovulation process, TGFB1, GDF9, $B M P 15, B M P 6$, and their receptor, $B M P R 2$, were assessed in the present study. TGF- $\beta 1$ (the protein product of TGFB1) is a factor that leads to cell division and differentiation [43]. Thus, based on scientific evidence, overexpression of TGF- $\beta 1$ can lead to increased amounts of stromal tissue or tissue fibrosis. As we found in the present study, the gene expression level of TGFB1 was decreased in PCO model animals. Histological assessments also showed that the stromal tissue surrounding cystic follicles accounted for the smaller size of ovaries in PCO animals. Recent evidence has indicated that expression of TGFB1 and subsequent alterations in the extracellular matrix might contribute to the pathogenesis of multicystic ovaries [44]. As the results showed, the gene expression of TGFB1 was significantly lower in the preantral follicles of the PCO group. However, on the contrary, Miao et al. [45] concluded that the TGF- $\beta 1$ pathway affected the expression of fibrillin 3 in stromal compartments of fetal ovaries, in which the ovarian stroma was larger. In some studies, elevated levels of TGF- $\beta 1$ have been seen in PCO, unlike the results of the present study. In the analysis of TGFB1 gene expression in various $\mathrm{PCO}$-affected preantral follicles, this difference can serve as a turning point. BMP signaling influences female fertility of different species in different ways. In this study, gene expression levels of BMP6 and BMP15, as well as their receptor (BMPR2), were measured. BMP- 6 is required for normal female fertility [46]. TGF- $\beta 1$ and BMP- 6 are primary mediators of ovarian follicular growth [47]. However, although no major variations in $B M P 6$ expression levels have been observed in the present sample, decreased levels of TGFB1 were found in preantral PCO follicles. The gene expression of GDF9 and BMP15 in PCO depends on the follicular stage [11]. We also found no major variations in BMP15 gene expression in preantral PCO follicles. BMP15 causes the transition from primordial follicles to primary and secondary follicles. Along with the results of our study, another study also suggested that BMP15 has no effects on the follicular transition into preovulatory follicles [48]. There is evidence that the BMPR2 signaling pathway is impaired in the case of PCOS, most likely due to hyperaldosteronism [49]. An analysis of gene expression patterns showed that $B M P R 2$ levels were significantly reduced. These results imply that PCO does not have any major effect on the expression of BMP6 or $B M P 15$, but their receptor is significantly affected. Thus, the probable incomplete function of BMP6 and BMP15 is related to their receptor. In many mammalian species, GDF9 is important for early follicular development through its direct action on granulosa cells, allowing proliferation and differentiation [50]. GDF9 is important for folliculogenesis, oogenesis, and ovulation and therefore plays a major role in women's fertility [51]. In this study, the expression level of GDF9 was 
lower in preantral PCOS follicles. Karagul et al. [6] also found reduced levels of GDF9 expression in primary follicles. Teixeira Filho et al. [52] also reported the aberrant expression of GDF9 in oocytes of women with PCOS. Overall, PCO disrupts the expression of key genes in folliculogenesis.

We concluded that the expression of the GDF9, TGFB1, and $B M P R 2$ genes in the preantral follicles of the PCO group was significantly reduced, resulting in impaired folliculogenesis and no ovulation. The expression of the BMP6 and BMP15 genes in both normal antral follicles and antral follicles in the PCO group was normal, with no significant changes. All sexual hormones were decreased, except for progesterone, which showed increased levels in the PCO group. Thus, unlike the hyperandrogenism that has been reported in other published papers, we found hyperprogesteronism in PCO-affected animals following EV injections. Although gene expression, histopathological alterations, and serum hormone levels were measured in the present study in an EV-induced PCO mouse model, it is recommended to design an experimental study on DHEA-induced PCO animals to observe molecular alterations.

\section{Conflict of interest}

No potential conflict of interest relevant to this article was reported.

\section{ORCID}

$\begin{array}{ll}\text { Reza Asghari } & \text { https://orcid.org/0000-0003-4791-4312 } \\ \text { Vahid Shokri-Asl } & \text { https://orcid.org/0000-0002-4452-0919 } \\ \text { Hanieh Rezaei } & \text { https://orcid.org/0000-0001-6696-5313 } \\ \text { Mahmood Tavallaie } & \text { https://orcid.org/0000-0003-0080-4298 } \\ \text { Mostafa Khafaei } & \text { https://orcid.org/0000-0001-5661-4544 } \\ \text { Amir Abdolmaleki } & \text { https://orcid.org/0000-0001-9541-8829 } \\ \text { Abbas Majdi Seghinsara } & \text { https://orcid.org/0000-0002-2609-9219 }\end{array}$

\section{Author contributions}

Conceptualizatid: AMS, Data curation: VSA, Formal analysis: MT, Funding acquisition: AMS, Methodology: AMS, Project administration: HR, Visualization: MK, Writing-original draft: AA. Writing-review \& editing: $\mathrm{RA}$.

\section{References}

1. Azziz R, Carmina E, Chen Z, Dunaif A, Laven JS, Legro RS, et al. Polycystic ovary syndrome. Nat Rev Dis Primers 2016;2:16057.

2. Teede H, Deeks A, Moran L. Polycystic ovary syndrome: a complex condition with psychological, reproductive and metabolic manifestations that impacts on health across the lifespan. BMC Med 2010;8:41

3. Huang A, Brennan K, Azziz R. Prevalence of hyperandrogenemia in the polycystic ovary syndrome diagnosed by the National Institutes of Health 1990 criteria. Fertil Steril 2010;93:1938-41.

4. Bani Mohammad M, Majdi Seghinsara A. Polycystic ovary syndrome (PCOS), diagnostic criteria, and AMH. Asian Pac J Cancer Prev 2017;18:17-21.

5. Erickson GF, Shimasaki S. The physiology of folliculogenesis: the role of novel growth factors. Fertil Steril 2001;76:943-9.

6. Karagul Ml, Aktas S, Coskun Yilmaz B, Yilmaz M, Orekici Temel G. GDF9 and BMP15 expressions and fine structure changes during folliculogenesis in polycystic ovary syndrome. Balkan Med J 2018;35:43-54.

7. Piotrowska H, Kempisty B, Sosinska P, Ciesiolka S, Bukowska D, Antosik P, et al. The role of TGF superfamily gene expression in the regulation of folliculogenesis and oogenesis in mammals: a review. Vet Med 2013;58:505-15.

8. Sanfins A, Rodrigues P, Albertini DF. GDF9 and BMP15 direct the follicle symphony. J Assist Reprod Genet 2018;35:1741-50.

9. Persani L, Rossetti R, Di Pasquale E, Cacciatore C, Fabre S. The fundamental role of bone morphogenetic protein 15 in ovarian function and its involvement in female fertility disorders. Hum Reprod Update 2014;20:869-83.

10. Shi J, Yoshino O, Osuga Y, Koga K, Hirota Y, Hirata T, et al. Bone morphogenetic protein- 6 stimulates gene expression of follicle-stimulating hormone receptor, inhibin/activin beta subunits, and anti-Mullerian hormone in human granulosa cells. Fertil Steril 2009;92:1794-8.

11. Wei LN, Huang R, Li LL, Fang C, Li Y, Liang XY. Reduced and delayed expression of GDF9 and BMP15 in ovarian tissues from women with polycystic ovary syndrome. J Assist Reprod Genet 2014;31: 1483-90.

12. Shi D, Vine DF. Animal models of polycystic ovary syndrome: a focused review of rodent models in relationship to clinical phenotypes and cardiometabolic risk. Fertil Steril 2012;98:185-93.

13. Ben-Or S. Morphological and functional development of the ovary of the mouse. I. Morphology and histochemistry of the developing ovary in normal conditions and after FSH treatment. J Embryol Exp Morphol 1963;11:1-11.

14. Makker A, Goel MM, Das V, Agarwal A. PI3K-Akt-mTOR and MAPK signaling pathways in polycystic ovarian syndrome, uterine leiomyomas and endometriosis: an update. Gynecol Endocrinol 2012:28:175-81.

15. Hu L, Zhang Y, Chen L, Zhou W, Wang Y, Wen J. MAPK and ERK polymorphisms are associated with PCOS risk in Chinese women. 
Oncotarget 2017:8:100261-8.

16. Franks S. Can animal models of PCOS help point the way towards early and effective therapeutic intervention in women with the syndrome? Endocrinology 2015;156:2371-3.

17. Maliqueo M, Benrick A, Stener-Victorin E. Rodent models of polycystic ovary syndrome: phenotypic presentation, pathophysiology, and the effects of different interventions. Semin Reprod Med 2014;32:183-93.

18. Divyashree S, Janhavi P, Ravindra PV, Muthukumar SP. Experimental models of polycystic ovary syndrome: an update. Life Sci 2019;237:116911.

19. Newbold RR, Jefferson WN, Padilla-Banks E. Prenatal exposure to bisphenol $A$ at environmentally relevant doses adversely affects the murine female reproductive tract later in life. Environ Health Perspect 2009;117:879-85.

20. Alonso-Magdalena P, Ropero AB, Carrera MP, Cederroth CR, Baquie M, Gauthier BR, et al. Pancreatic insulin content regulation by the estrogen receptor ER alpha. PLoS One 2008;3:e2069.

21. Davis BJ, Maronpot RR, Heindel JJ. Di-(2-ethylhexyl) phthalate suppresses estradiol and ovulation in cycling rats. Toxicol Appl Pharmacol 1994;128:216-23.

22. Decherf S, Demeneix BA. The obesogen hypothesis: a shift of focus from the periphery to the hypothalamus. J Toxicol Environ Health B Crit Rev 2011;14:423-48.

23. Grun F, Blumberg B. Environmental obesogens: organotins and endocrine disruption via nuclear receptor signaling. Endocrinology 2006;147(6 Suppl):S50-5.

24. Wu XY, Li ZL, Wu CY, Liu YM, Lin H, Wang SH, et al. Endocrine traits of polycystic ovary syndrome in prenatally androgenized female Sprague-Dawley rats. Endocr J 2010;57:201-9.

25. Poornima R, Saranya P, Bhuvaneshwari S, Averal HI. Evaluation of Pergularia daemia and metformin in the treatment of PCOS in testosterone propionate induced albino wistar rats (Rattus norvegicus). Int J Sci Res Pub 2015;5:503-9.

26. Mahesh VB, Greenblatt RB. Isolation of dehydroepiandrosterone and 17alpha-hydroxy-delta5-pregenolone from the polycystic ovaries of the Stein-Leventhal syndrome. J Clin Endocrinol Metab 1962;22:441-8.

27. Kim EJ, Jang M, Choi JH, Park KS, Cho IH. An improved dehydroepiandrosterone-induced rat model of polycystic ovary syndrome (PCOS): post-pubertal improve PCOS's features. Front Endocrinol (Lausanne) 2018;9:735.

28. Manneras L, Cajander S, Holmang A, Seleskovic Z, Lystig T, Lonn M, et al. A new rat model exhibiting both ovarian and metabolic characteristics of polycystic ovary syndrome. Endocrinology 2007;148:3781-91.

29. van Houten EL, Kramer P, McLuskey A, Karels B, Themmen AP, Viss- er JA. Reproductive and metabolic phenotype of a mouse model of PCOS. Endocrinology 2012;153:2861-9.

30. Johansson J, Feng Y, Shao R, Lonn M, Billig H, Stener-Victorin E. Intense electroacupuncture normalizes insulin sensitivity, increases muscle GLUT4 content, and improves lipid profile in a rat model of polycystic ovary syndrome. Am J Physiol Endocrinol Metab 2010;299:E551-9.

31. Pinilla L, Trimino E, Garnelo P, Bellido C, Aguilar R, Gaytan F, et al. Changes in pituitary secretion during the early postnatal period and anovulatory syndrome induced by neonatal oestrogen or androgen in rats. J Reprod Fertil 1993;97:13-20.

32. Stener-Victorin E, Ploj K, Larsson BM, Holmang A. Rats with steroid-induced polycystic ovaries develop hypertension and increased sympathetic nervous system activity. Reprod Biol Endocrinol 2005;3:44.

33. Norman RJ, Noakes M, Wu R, Davies MJ, Moran L, Wang JX. Improving reproductive performance in overweight/obese women with effective weight management. Hum Reprod Update 2004;10:267-80.

34. Ikeda K, Baba T, Morishita M, Honnma H, Endo T, Kiya T, et al. Longterm treatment with dehydroepiandrosterone may lead to follicular atresia through interaction with anti-Mullerian hormone. J Ovarian Res 2014;7:46.

35. Mesbah F, Moslem M, Vojdani Z, Mirkhani H. Estradiol valerate-induced polycystic ovary syndrome: an animal model study. Armaghane Danesh 2011;15:325-34.

36. Franks S, Hardy K. Folliculogenesis in polycystic ovaries. In: Dunaif A, Chang RJ, Franks S, Legro RS, editors. Polycystic ovary syndrome: current controversies, from the ovary to the pancreas. Totowa: Humana Press; 2008. p. 1-7.

37. Brawer JR, Munoz M, Farookhi R. Development of the polycystic ovarian condition (PCO) in the estradiol valerate-treated rat. Biol Reprod 1986;35:647-55.

38. Haggstrom M. Reference ranges for estradiol, progesterone, luteinizing hormone and follicle-stimulating hormone during the menstrual cycle. Wiki J Med 2014;1:1-5.

39. Miri M, Karimi Jashni H, Alipour F. Effect of exercise intensity on weight changes and sexual hormones (androstenedione and free testosterone) in female rats with estradiol valerate-induced PCOS. J Ovarian Res 2014;7:37.

40. Pournaderi PS, Yaghmaei P, Khodaei H, Noormohammadi Z, Hejazi $\mathrm{SH}$. The effects of 6-Gingerol on reproductive improvement, liver functioning and Cyclooxygenase-2 gene expression in estradiol valerate: induced polycystic ovary syndrome in Wistar rats. Biochem Biophys Res Commun 2017;484:461-6.

41. Venegas B, De Leon Gordillo LY, Rosas G, Espinoza JA, Moran C, Dominguez $\mathrm{R}$, et al. In rats with estradiol valerate-induced poly- 
cystic ovary syndrome, the acute blockade of ovarian $\beta$-adrenoreceptors improve ovulation. Reprod Biol Endocrinol 2019;17:95.

42. Laird M, Thomson K, Fenwick M, Mora J, Franks S, Hardy K. Androgen stimulates growth of mouse preantral follicles in vitro: interaction with follicle-stimulating hormone and with growth factors of the TGF $\beta$ superfamily. Endocrinology 2017;158:920-35.

43. Ghadami M, Makita Y, Yoshida K, Nishimura G, Fukushima Y, Wakui $\mathrm{K}$, et al. Genetic mapping of the Camurati-Engelmann disease locus to chromosome 19q13.1-q13.3. Am J Hum Genet 2000;66: 143-7.

44. Raja-Khan N, Urbanek M, Rodgers RJ, Legro RS. The role of TGF- $\beta$ in polycystic ovary syndrome. Reprod Sci 2014;21:20-31.

45. Miao Zl, Wang ZN, Yang YD, Chen LQ, Rong CU, Wang XL, et al. Role of TGF- $\beta 1$ in the formation of ovarian interstitial fibrosis in PCOS rat. J Reprod Contracept 2008;19:83-92.

46. Sugiura K, Su YQ, Eppig JJ. Does bone morphogenetic protein 6 (BMP6) affect female fertility in the mouse? Biol Reprod 2010;83: 997-1004.

47. Zhang XY, Chang HM, Zhu H, Liu RZ, Leung PC. BMP6 increases TGF- $\beta 1$ production by up-regulating furin expression in human granulosa-lutein cells. Cell Signal 2019;55:109-18.

48. Moore RK, Shimasaki S. Molecular biology and physiological role of the oocyte factor, BMP15. Mol Cell Endocrinol 2005;234:67-73.

49. de Resende LO, Vireque AA, Santana LF, Moreno DA, de Sa Rosa e Silva AC, Ferriani RA, et al. Single-cell expression analysis of BMP15 and GDF9in mature oocytes and BMPR2 in cumulus cells of women with polycystic ovary syndrome undergoing controlled ovarian hyperstimulation. J Assist Reprod Genet 2012;29: 1057-65.

50. Otsuka F, McTavish KJ, Shimasaki S. Integral role of GDF9 and BMP15 in ovarian function. Mol Reprod Dev 2011;78:9-21.

51. de Castro FC, Cruz MH, Leal CL. Role of growth differentiation factor 9 and bone morphogenetic protein 15 in ovarian function and their importance in mammalian female fertility: a review. Asian-Australas J Anim Sci 2016;29:1065-74.

52. Teixeira Filho FL, Baracat EC, Lee TH, Suh CS, Matsui M, Chang RJ, et al. Aberrant expression of growth differentiation factor-9 in oocytes of women with polycystic ovary syndrome. J Clin Endocrinol Metab 2002;87:1337-44. 